\title{
Recherche de la résistance des strongles aux anthelminthiques chez le mouton en Algérie
}

\author{
A. Boulkaboul ${ }^{1 *}$ A. Boucif ${ }^{1}$ K. Senouci ${ }^{2}$
}

Mots-clés

Ovin - Marshallagia marshalli Helminthe - Résistance aux anthelminthiques - Algérie.

\begin{abstract}
Résumé
Une étude de la résistance des strongles gastro-intestinaux aux anthelminthiques chez le mouton a été menée dans une région semi-aride d'Algérie. Deux anthelminthiques (albendazole et ivermectine) communément utilisés ont été testés respectivement sur 120 et 40 animaux dans 14 fermes par le test de réduction de l'excrétion fécale des œufs (Fecrt). Les résultats ont révélé une grande efficacité de l'ivermectine (> $95 \%$ ) à la dose de $0,2 \mathrm{mg} / \mathrm{kg}$ de poids vif. L'efficacité globale de l'albendazole à la dose de $5 \mathrm{mg} / \mathrm{kg}$ de poids vif a été de 94,2 p. 100, mais elle a été faible $(<90 \%)$ dans quatre à cinq fermes sur dix, et la réduction de l'excrétion fécale des œufs a été comprise entre 83,3 et 100 p. 100. La persistance de Marshallagia marshalli après traitement a été significativement la plus forte $(70,4 \%)$ parmi d'autres strongles gastrointestinaux. L'interprétation du Fecrt à faible nombre d'œufs par gramme de fèces liée à cette persistance et son rapport avec la présence annuelle de l'espèce chez les ovins ont été analysés.
\end{abstract}

\section{INTRODUCTION}

Les strongles gastro-intestinaux (SGI) sont des parasites communs chez les ruminants, fréquemment impliqués dans des pertes économiques notables dans les élevages (15). L'usage démesuré des produits anthelminthiques a entraîné l'apparition d'une chimiorésistance au niveau mondial (11). En Afrique, la lutte contre ces parasites rencontre des problèmes de disponibilité et de coût des produits. Il y a ainsi souvent recours à des génériques bon marché et de qualité douteuse $(3,16)$ contribuant au développement de la résistance des parasites signalée sur le continent (22). Au Maghreb, des cas de résistance ont été rapportés chez les ovins au Maroc (4), puis en Algérie dans la zone Est (2).

Dans une étude antérieure des auteurs effectuée dans le sud-ouest de l'Algérie où l'élevage ovin est très important (6), le parasitisme gastro-intestinal est caractérisé par une forte fréquence des genres Marshallagia et Nematodirus, peu prolifiques, dont le nombre d'œufs par gramme de fèces (opg) est souvent bas, rendant difficile l'interprétation du test de réduction de l'excrétion fécale des œufs (Fecrt) par les formules conventionnelles (7). Le but de cette étude a été la détection de la résistance des SGI aux anthelminthiques par le

1. Laboratoire de parasitologie, département des Sciences vétérinaires, Université Ibn Khaldoun, Tiaret, Algérie.

2. Faculté de Biologie, Université Es-Senia, Oran, Algérie.

* Auteur pour la correspondance

2, rue du Vieux Palais, Alger 16000, Algérie.

E-mail : b_boulkaboul@yahoo.fr
Fecrt, en incluant le taux de persistance des œufs des strongles chez les moutons après traitement comme paramètre complémentaire.

\section{MATERIEL ET METHODES}

\section{Zone d'étude}

L'étude a été réalisée dans une région semi-aride du Sud-Ouest d'Algérie (Tiaret) à 34-35 $5^{\circ}$ de lat. $\mathrm{N}$ et $0-2^{\circ}$ de long. E, à $1000 \mathrm{~m}$ d'altitude, et composée de vastes plaines pour la céréaliculture au Nord et d'une grande steppe au Sud. Elle est caractérisée par un climat chaud et sec en été, très froid en hiver, et par une pluviométrie annuelle inférieure à $500 \mathrm{~mm}$.

\section{Animaux \\ Au total, 272 moutons des deux sexes âgés de 6 à 14 mois, non traités depuis deux mois au minimum, ont été choisis au hasard dans des troupeaux appartenant à des éleveurs privés. Les fermes étaient réparties dans six communes de Tiaret dans un rayon de $70 \mathrm{~km}$ (tableau I). Les élevages étaient traditionnels et extensifs, et les animaux (entre 20 et 200) étaient conduits librement sur des parcours steppiques, des prairies à céréales, ou parfois en bordure des maquis selon la localisation des fermes. En hiver, ils étaient nourris au fourrage sec et les brebis recevaient un supplément d'orge à la période de lutte. \\ Les éleveurs faisaient des transactions dans des marchés à bestiaux hebdomadaires, mais les sujets nouvellement acquis ne subissaient pas de quarantaine. Ils se fournissaient en anthelminthiques auprès}


des vétérinaires ou directement auprès de sociétés privées de vente de médicaments, préférant des génériques pour leur moindre coût.

Le déparasitage des moutons était souvent pratiqué avec l'albendazole, deux fois par an (mars et octobre), dans la plupart des fermes, et moins fréquemment avec l'ivermectine, en particulier contre les ectoparasites. Ces deux anthelminthiques ont été choisis pour l'étude.

Les sujets ont été bouclés avant de procéder aux prélèvements fécaux individuels. Ils n'ont pas été sélectionnés pour leurs niveaux d'opg. Dans chaque ferme, les moutons ont été gardés en bergerie pendant l'étude.

\section{Traitements anthelminthiques}

Les traitements ont été effectués sans diète préalable. L'essai de l'ivermectine a été réalisé dans quatre fermes (A, B, C et $\mathrm{D})$, avec deux lots de 10 animaux chacun (l'un traité, l'autre témoin) (tableau II). Le produit (Baymec, Bayer, Allemagne) a été administré à la dose de $0,2 \mathrm{mg} / \mathrm{kg}$ de poids vif par injection sous-cutanée.

\section{Tableau I}

Distribution des fermes étudiées dans la région de Tiaret (Algérie) et utilisation des anthelminthiques chez les ovins

\begin{tabular}{|c|c|c|c|c|}
\hline Commune & Zone & Ferme & $\begin{array}{l}\text { Nb. traitements/an } \\
\text { Albendazole }\end{array}$ & $\begin{array}{l}\text { Nb. traitements /an } \\
\text { Ivermectine }\end{array}$ \\
\hline \multirow[t]{3}{*}{ Tiaret } & Plaine & A & 2 (mars, octobre) depuis 6 ans & 2 (mars, octobre) depuis 6 ans \\
\hline & Maquis & B & 2 (mars, octobre) & 2 (mars, octobre) \\
\hline & & 1 & 3 (mars, juin, octobre) depuis 4 ans & 2 (mars, juin) depuis 3 ans \\
\hline \multirow[t]{3}{*}{ Sougueur } & Plaine & $\mathrm{C}$ & 2 (mars, octobre) & 2 (mars, octobre) \\
\hline & & $\mathrm{D}$ & 2 (mars, octobre) & 1 (juin) \\
\hline & & 2 & 3 (variable) & 1 (juin) \\
\hline \multirow[t]{2}{*}{ Frenda } & Plaine & 3 & 2 (mars, octobre) & 2 (mars, octobre) \\
\hline & & 4 & 2 (mars, octobre) depuis 6 ans & 2 (mars, octobre) depuis 4 ans \\
\hline \multirow[t]{2}{*}{ Mahdia } & Plaine & 5 & 3 (mars, juin, octobre) depuis 10 ans & 2 (mars, octobre) depuis 7 ans \\
\hline & & 6 & 2 (mars, octobre) & 1 (juin) \\
\hline \multirow[t]{2}{*}{ Ain Dheb } & Steppe & 7 & 3 (variable) & 1 (juillet) \\
\hline & & 8 & 2 (variable) & 0 \\
\hline \multirow[t]{2}{*}{ Guertoufa } & Maquis & 9 & 2 (variable) depuis 4 ans & 1 (juillet) depuis 4 ans \\
\hline & & 10 & 2 (mars, octobre) & 1 (juillet) \\
\hline
\end{tabular}

\section{Tableau II}

Efficacité de l'ivermectine contre les strongles gastro-intestinaux chez des ovins naturellement infestés dans la région de Tiaret (Algérie), par le Fecrt *

\begin{tabular}{|c|c|c|c|c|c|c|c|c|}
\hline \multirow[b]{2}{*}{ Ferme } & \multicolumn{8}{|c|}{ Ivermectine $(0,2 \mathrm{mg} / \mathrm{kg})$ en injection } \\
\hline & $\begin{array}{c}\text { MC1 } \\
\text { (n) }\end{array}$ & $\begin{array}{c}\text { MT1 } \\
(n)\end{array}$ & $\begin{array}{c}\text { Fecr (a) } \\
(\%)\end{array}$ & $\begin{array}{c}\text { Fecr (b) } \\
\quad(\%)\end{array}$ & $\begin{array}{c}\text { Fecr }(c) \\
(\%)\end{array}$ & $\begin{array}{c}\text { Fecr (d) } \\
(\%)\end{array}$ & $\begin{array}{c}\text { Fecr (e) } \\
(\%)\end{array}$ & $\begin{array}{c}\text { Diagnostic par Fecr4 } \\
\text { IC } 95 \% \\
\text { Limite inférieure }\end{array}$ \\
\hline A & $270(10)$ & 305 (10) & 97,1 & 96,7 & 97,5 & 96,2 & 98,7 & $93(\mathrm{~S})$ \\
\hline B & $325(10)$ & $340(10)$ & 99,3 & 99,2 & 99,4 & 99,2 & 99,5 & $95(\mathrm{~S})$ \\
\hline C & $350(10)$ & $380(10)$ & 98,9 & 98,6 & 99 & 98,5 & 99,6 & $93(\mathrm{~S})$ \\
\hline $\mathrm{D}$ & 395 (10) & $620(10)$ & 97,1 & 97,5 & 98,2 & 96,2 & 99,4 & $91(\mathrm{~S})$ \\
\hline Total & $335(40)$ & $411,2(40)$ & 98,1 & 97,4 & 98,6 & 97,5 & 99,3 & \\
\hline
\end{tabular}

* Test de réduction de l'excrétion fécale

Opg : œufs/g de fèces

Fecr4 : logiciel pour la détection de la résistance aux anthelminthiques par Fecrt

$\mathrm{MC}_{1}$ : moyenne d'opg à $\mathrm{J} 0$ chez les témoins non traités.

$\mathrm{MT}_{1}$ : moyenne d'opg avant traitement chez les sujets à traiter.

(Cameron, U of Sidney, 2004)

IC : intervalle de confiance

Fecr : (a) selon Coles et coll. (Vet. Parasitol., 1992) ; (b) selon Kochapakdee et coll.

(Vet.Rec., 1995) ; (c) selon Dash et coll. (Aust. Vet.J., 1988) ; (d) : selon Powers et

coll. (Vet.Parasitol., 1982); (e) selon Presidente (CSIRO, 1985). 
L'essai de l'albendazole a été réalisé dans dix fermes (de 1 à 10), avec deux lots de 12 animaux chacun (l'un traité, l'autre témoin) (tableau III). L'absence de lot témoin dans quatre fermes (7 à 10) a été imposée par les propriétaires. Le produit (Albendavet 2,5\%, Invesa, Espagne) a été administré par voie orale à la dose de $5 \mathrm{mg} / \mathrm{kg}$ de poids vif.

\section{Analyses parasitologiques et statistiques}

Chaque animal a subi deux collectes de fèces, avant (jour 0 ) et après traitement : à 10 jours pour l'albendazole et à 14 jours pour l'ivermectine, selon Coles et coll. (11). Les échantillons fécaux ont été transportés dans des sacs en plastique en glacière au laboratoire de l'université pour analyse. La coproscopie a été faite par la méthode de flottaison en solution de chlorure du sodium saturée (20). Le dénombrement d'œufs de strongles, incluant ceux de Nematodirus et Marshallagia, a été réalisé à l'aide d'une lame McMaster à deux chambres (VWR international) au seuil minimal de 25 opg.

La réduction de l'excrétion fécale des œufs (Fecr) a été basée selon les formules suivantes :

- Fecr $(\%)=1-\left(\mathrm{MT}_{2} / \mathrm{MC}_{2}\right) \times 100$, selon Coles et coll. (10) ;

- Fecr $(\%)=1-\left(\mathrm{MT}_{2} / \mathrm{MT}_{1}\right)$ x 100, selon Kochapakdee et coll. (14) ;

- Fecr $(\%)=1-\left[\left(\mathrm{MT}_{2} / \mathrm{MT}_{1}\right) \times\left(\mathrm{MC}_{1} / \mathrm{MC}_{2}\right)\right] \times 100$, selon Dash et coll. (12) ;

- Fecr $(\%)=1-\left(\mathrm{MT}_{2} / \mathrm{MC}_{1}\right) \times 100$, selon Powers et coll. (18);

$-\operatorname{Fecr}(\%)=1-\left[\left(\mathrm{MT}_{2} / \mathrm{MT}_{1}\right) \times\left(\mathrm{MC}_{1} / \mathrm{MC}_{2}\right)\right] \times 100$, selon Presidente (19)

où $\mathrm{MT}_{1}$ est la moyenne de l'opg chez les moutons à traiter à jour 0 (avant traitement), $\mathrm{MT}_{2}$ la moyenne de l'opg chez les moutons traités après 10-14 jours, $\mathrm{MC}_{1}$ la moyenne de l'opg chez les témoins non traités à jour $0, \mathrm{MC}_{2}$ la moyenne de l'opg chez les témoins non traités après 10-14 jours. Seule la méthode de Presidente (19) utilise les moyennes géométriques, les autres étant basées sur les moyennes arithmétiques.

La persistance des parasites après traitement, toutes fermes confondues, a été évaluée en terme de pourcentage :

$\mathrm{P}(\%)=\mathrm{N}_{2}$ (somme des sujets parasités après traitement) / $\mathrm{N}_{1}$ (somme des sujets parasités avant traitement) x 100 .

L'analyse des données a été faite avec le logiciel Statistica (Statsoft, USA) pour les statistiques descriptives et les tests de signification (comparaison des moyennes, des pourcentages et test $t$ ), et par le programme Fecr4 (8) qui utilise le seuil de 95 p. 100 de réduction de l'excrétion fécale et l'intervalle de confiance inférieur à 90 p. 100 pour déterminer la résistance (10). Les valeurs $\mathrm{P}<0,05$ ont été considérées comme significatives.

\section{RESULTATS}

La coproscopie a révélé la présence d'œufs de Marshallagia marshalli, de Nematodirus spp. et d'autres strongles gastro-intestinaux (ASGI), ainsi que ceux de Moniezia expansa et M. benedeni. Initialement, les valeurs individuelles d'opg des strongles chez les moutons étaient comprises entre 50 et 5 000, mais sont toujours restées inférieures à 400 pour $M$. marshalli et Nematodirus spp.

L'efficacité globale de l'ivermectine a été de 97,4 à 98,6 p. 100, avec une réduction fécale des œufs supérieure à 95 p. 100 dans toutes les fermes (tableau II). Parmi onze moutons infestés par M. marshalli, il y a eu seulement deux cas de persistance des œufs après traitement $(18,1$ p. 100). La persistance des œufs de Nematodirus spp. et des ASGI a été respectivement de 20 et 17,3 p. 100.

\section{Tableau III}

Efficacité de I'albendazole contre les strongles gastro-intestinaux chez des ovins naturellement infestés dans la région de Tiaret (Algérie), par le Fecrt*

\begin{tabular}{|c|c|c|c|c|c|c|c|c|}
\hline \multirow[b]{2}{*}{ Ferme } & \multicolumn{7}{|c|}{ Albendazole $(5 \mathrm{mg} / \mathrm{kg})$ per os } & \multirow[b]{2}{*}{$\begin{array}{c}\text { Diagnostic par Fecr4 } \\
\text { IC } 95 \% \\
\text { Limite inférieure }\end{array}$} \\
\hline & $\begin{array}{c}\text { MC1 } \\
\text { (n) }\end{array}$ & $\begin{array}{c}\text { MT1 } \\
\text { (n) }\end{array}$ & $\begin{array}{c}\text { Fecr (a) } \\
(\%)\end{array}$ & $\begin{array}{c}\text { Fecr (b) } \\
\quad(\%)\end{array}$ & $\begin{array}{c}\text { Fecr }(c) \\
(\%)\end{array}$ & $\begin{array}{c}\text { Fecr }(d) \\
\quad(\%)\end{array}$ & $\begin{array}{c}\text { Fecr }(\mathbf{e}) \\
(\%)\end{array}$ & \\
\hline 1 & $987,5(12)$ & $1387,5(12)$ & 98,6 & 98,8 & 99 & 98,3 & 99,7 & $94(S)$ \\
\hline 2 & $391,6(12)$ & $454,1(12)$ & 96,8 & 95,4 & 97,3 & 94,6 & 99,3 & 87 (FR) \\
\hline 3 & $508,3(12)$ & $595,8(12)$ & 97,2 & 97,2 & 97,7 & 96,7 & 99,4 & $90(\mathrm{~S})$ \\
\hline 4 & $370,8(12)$ & $383,3(12)$ & 89,2 & 89,6 & 89,6 & 89,3 & 97,4 & $53(\mathrm{R})$ \\
\hline 5 & $195,8(12)$ & $195,8(12)$ & 86,8 & 84 & 86,9 & 84 & 88,9 & $77(\mathrm{R})$ \\
\hline 6 & $183,3(12)$ & $137,5(12)$ & 86,9 & 83,3 & 82,7 & 87,5 & 87,5 & $69(\mathrm{R})$ \\
\hline 7 & - & $112,5(12)$ & - & 83,3 & - & - & - & $63(\mathrm{R})$ \\
\hline 8 & - & $95,8(12)$ & - & 100 & - & - & - & $100(\mathrm{~S})$ \\
\hline 9 & - & $325(12)$ & - & 90,4 & - & - & - & $79(\mathrm{R})$ \\
\hline 10 & - & $287,5(12)$ & - & 89,1 & - & - & - & $83(R)$ \\
\hline Total & & $397,5(120)$ & & 94,2 & & & & \\
\hline \multicolumn{5}{|c|}{$\begin{array}{l}\text { * Test de réduction de l'excrétion fécale } \\
\text { Opg : œufs/g de fèces } \\
\mathrm{MC}_{1} \text { : moyenne d'opg à J0 chez les témoins non traités. } \\
\mathrm{MT}_{1} \text { : moyenne d'opg avant traitement chez les sujets à traiter. } \\
\text { Fecr : (a) selon Coles et coll. (Vet. Parasitol., 1992); (b) selon Kochapakdee et coll. } \\
\text { (Vet. Rec, 1995); (c) selon Dash et coll. (Aust. Vet. J., 1988); (d) : selon Powers et } \\
\text { coll. (Vet. Parasitol., 1982); (e) selon Presidente (CSIRO, 1985). }\end{array}$} & \multicolumn{4}{|c|}{$\begin{array}{l}\text { Fecr } 4 \text { : logiciel pour la détection de la résistance aux anthelminthiques par Fecrt } \\
\text { (Cameron, 2004, U of Sidney). } \\
\text { IC : intervalle de confiance } \\
\mathrm{S}: \text { sensible ; R : résistant ; FR : faiblement résistant }\end{array}$} \\
\hline
\end{tabular}


L'efficacité globale de l'albendazole a été de 94,2 p. 100, selon la méthode de Kochapakdee et coll. (14). La réduction fécale des œufs a été comprise entre 83,3 et 100 p. 100, et a été inférieure à 90 p. 100 dans cinq fermes (tableau III). Le diagnostic de faible résistance dans la ferme 2 par le Fecr4, et ce malgré une réduction supérieure à 95 p. 100, a été lié à un intervalle de confiance inférieur à 90 p. 100.

Parmi 120 moutons traités, malgré une prévalence plus faible (36,6 p. 100), M. marshalli a été plus persistant après traitement que Nematodirus spp. et les ASGI (figure 1). Une disparition totale des œufs de Moniezia a été observée après traitement avec l'albendazole.

\section{DISCUSSION}

Habituellement, quand la résistance est déclarée, les niveaux d'opg des SGI restent élevés (5), mais dans le cas de Marshallagia et Nematodirus, ils sont initialement très bas et donc la présence d'une résistance passe facilement inaperçue.

Dans une étude antérieure des auteurs (6), la plupart des strongles gastro-intestinaux du mouton avaient une évolution saisonnière, alors que Marshallagia sp. et Nematodirus spp. étaient présents avec un même niveau durant toute l'année.

M. marshalli, bien que présent sur différents continents, n'est pas aussi souvent cité dans les strongyloses digestives ovines que Haemonchus, Trichostrongylus ou Nematodirus. D'après Oripov (17), l'espèce serait à l'origine d'anémie, d'émaciation, de déplétion vitaminique et d'une inflammation locale aiguë. Cependant, un essai expérimental à la dose de 10000 larves/mouton n'a pas entraîné d'effets pathogènes (1).

Les benzimidazoles et les ivermectines sont, pour leur bonne efficacité, largement utilisés pour lutter contre les SGI. D'après van Schalkwyk et coll. (21), l'efficacité de l'albendazole à la dose de $3,8 \mathrm{mg} / \mathrm{kg}$ est supérieure à 98,8 p. 100 contre Haemonchus, Ostertagia, Trichostrongylus, Nematodirus, Marshallagia et Chabertia. Wescott et LeaMaster (23) rapportent une efficacité de l'ivermectine supérieure à 96 p. 100 contre les SGI du mouton, y compris M. marshalli.

L'espèce la plus fréquemment citée comme résistante aux anthelminthiques est $H$. contortus, soumise, en raison de sa forte

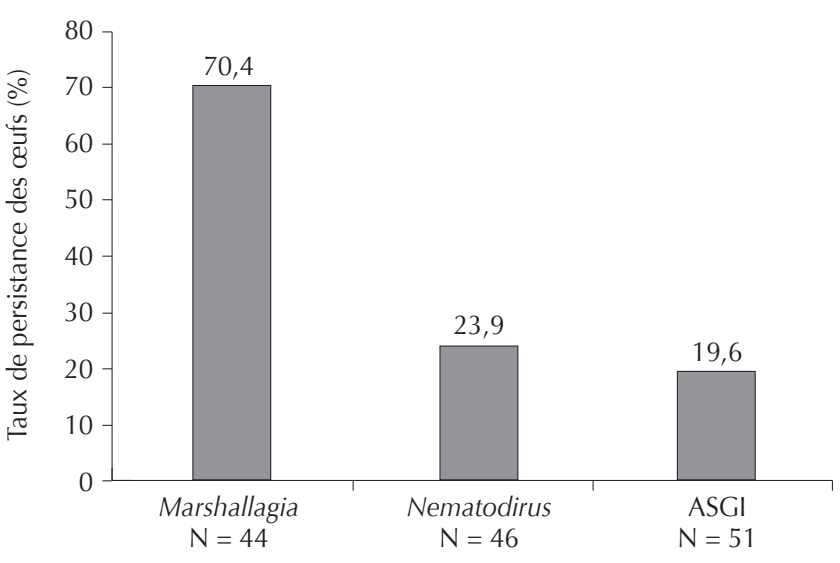

Figure 1 : taux de persistance des œufs de strongles gastrointestinaux après traitement avec l'albendazole $(5 \mathrm{mg} / \mathrm{kg}$ de poids vif, per os) de 120 moutons naturellement infestés. $N$ : nombre de moutons infestés avant traitement. ASGI : autres strongles gastro-intestinaux. prolificité et de son cycle court, à une forte pression de sélection. Cependant, plus récemment, la résistance aux benzimidazoles a été signalée chez Nematodirus spathiger (9) et M. marshalli (13). Bentounsi et coll. (3) rapportent une résistance à l'albendazole chez les strongles Teladorsagia, Trichostrongylus, Marshallagia et Nematodirus, dans cinq fermes pilotes à l'est de l'Algérie.

Dans la présente étude, le Fecrt basé sur l'opg relatif aux SGI a donné une efficacité globale élevée pour l'ivermectine, mais plus faible pour l'albendazole (83,3 à 100 p. 100). D'après la limite de 95 p. 100 du Fecrt, la résistance contre l'albendazole a été présente dans six fermes. Mais selon la formule de Presidente (19) la ferme 4 n'a pas été concernée.

La forte réduction fécale des œufs dans les fermes 1 et 3 , où les opg étaient les plus importants (maximum respectivement de 1500 et 5000 ), suggère que la qualité du produit n'était pas impliquée dans le cas des faibles réductions observées dans les autres fermes. En revanche, les éleveurs ont utilisé les produits de façon désordonnée et ont augmenté les doses de l'albendazole pour atteindre les strongles respiratoires sur recommandation des vétérinaires. La présence de $M$. marshalli a dû causer un biais dans le Fecrt, du fait d'opg toujours inférieurs à $500(2,5)$. En omettant les œufs de l'espèce, l'efficacité globale de l'albendazole a atteint 95,6 p. 100.

L'évaluation du taux global de persistance des œufs des SGI après traitement avec l'albendazole a révélé une valeur élevée pour $M$. marshalli, avec une différence très significative par rapport à Nematodirus et aux ASGI $(\mathrm{p}<0,05)$ (figure 1).

Malgré la présence de $M$. marshalli avant traitement par l'ivermectine chez les moutons, le Fecrt a révélé une efficacité globale supérieure à 95 p. 100. Le programme Fecr4 a également montré une résistance contre l'albendazole semblant liée à $M$. marshalli dans certaines des fermes contrôlées. Ceci doit être confirmé par nécropsie, tests in vitro ou suivi coproscopique plus prolongé.

En pratique, le vétérinaire peut utiliser une coproscopie qualitative rapide. Du fait de l'interprétation délicate du Fecrt dans le cas de faibles opg, l'observation d'une fréquence élevée de la persistance des œufs des parasites après traitement pourrait servir d'indice révélateur du développement d'une résistance aux anthelminthiques dans un cheptel ovin donné.

La forte persistance notée pour $M$. marshalli dans la région étudiée pourrait en partie expliquer le taux d'infestation important des ovins par cette espèce pendant l'année. Il serait donc intéressant, dans les zones endémiques, de vérifier son implication pathologique dans les strongyloses digestives ovines.

\section{BIBLIOGRAPHIE}

1. AL-KHALIDI N.W., ARSALAN S.H., MUSLIH N.J., AL-SAAD K.M., 1989. Pathogenicity of Marshallagia marshalli (Ransom) in sheep. Indian J. Anim. Sci., 59: 917-919.

2. BENTOUNSI B., ATTIR B., MERADI S., CABARET J., 2007. Repeated faecal egg counts to identify gastrointestinal nematode resistance in a context of low-level infection of sheep on farms in Eastern Algeria. Vet. Parasitol., 144: 104-110.

3. BENTOUNSI B., ZOUIOUECH H., BENCHIKH-ELFEGOUN C., KOHIL K., BOUZEKRI M., CABARET J., 2003. Efficacité comparée des spécialités d'albendazole distribuées en Algérie. Revue Méd. vét., 154 : 649-652.

4. BERRAG B., OUKESSOU M., CABARET J., 2002. Résistance des nématodes gastro-intestinaux des ovins et des caprins aux benzimidazoles au Maroc. Animalis, 1 : 25-28.

5. BOULKABOUL A., BOUAKKAZ A., KERBCEUF D., 2006. Détection d'une résistance aux benzimidazoles chez les strongles digestifs du cheval en Algérie. Revue Méd. vét., 157 : 59-64. 
6. BOUlKabOUL A., MOULAYE K., 2006. Parasitisme interne du mouton de race Ouled Djellal en zone semi-aride d'Algérie. Revue Elev. Méd. vét. Pays trop., 59 : 23-29.

7. CABARET J., 2004. Efficacy evaluation of anthelmintics: which methods to use in the field? Parasitologia, 46: 241-243.

8. CAMERON A., 2004. FECR4: Egg count reduction analysis. University of Sydney, Australia, AusVet, Animal Health Services. http://sydney.edu. $\mathrm{au} /$ vetscience/sheepwormcontrol/software/index.html

9. CHALMERS K., 1985. Detection of benzimidazole resistant Nematodirus spathiger. N. Z. vet. J., 33: 53.

10. COLES G.C., BAUER C., BORGSTEED F.H.M., GEERTS S., KLEI T.R., TAYLOR M.A, WALLER P.J., 1992. Methods of detection of anthelmintic resistance in nematodes of veterinary importance. Vet. Parasitol., 44: 35-44.

11. COLES G.C., JACKSON F., POMROY W.E., PRICHARD R.K., VON SAMSON-HIMMELSTJERNA G., WOODLAND A., TAYLOR M.A., VERCRUYSSE J., 2006. The detection of anthelmintic resistance in nematodes of veterinary importance. Vet. Parasitol., 136: 167-185.

12. DASH K., HALL K., BARGER I.A., 1988. The role of arithmetic and geometric worm egg counts in faecal egg count reduction test and in monitoring strategic drenching programs in sheep. Aust. Vet. J., 65: 66-68.

13. GHOLAMIAN A., GALEHDARI H., ESLAMI A., NABAVI L., 2007. Study of $\beta$-tubulin gene polymorphisms in Haemonchus contortus isolated from sheep populations in Khouzestan, Southwestern Iran. Iran. J. Vet. Res., 8: 239-243.

14. KOCHAPAKDEE S., PANDEY V.S., PRALOMKANN W., CHOLDUMRONGKUL S., NGAMPONGSAI W., LAWPETCHARA A., 1995. Anthelmintic resistance in goats in Southern Thailand. Vet. Rec., 137: $124-125$

\section{Summary}

Boulkaboul A., Boucif A., Senouci K. Research of gastro-intestinal nematode resistance to anthelmintics in sheep in Algeria

A study on gastro-intestinal nematode resistance to anthelmintics in sheep was carried out in a semiarid area of Algeria. Two drugs (albendazole and ivermectin) commonly used were tested in 120 and 40 sheep, respectively, in 14 farms by the fecal egg count reduction test (FECRT). Results revealed high efficiency of ivermectin (>95\%) at the dose of $0.2 \mathrm{mg} / \mathrm{kg}$ body weight. Overall efficiency of albendazole at $5 \mathrm{mg} / \mathrm{kg}$ was $94.2 \%$, but it was low $(<90 \%)$ in four to five farms out of ten, and fecal egg reduction reached $83.3-100 \%$. The persistence of Marshallagia marshalli after treatment was significantly the highest $(70.4 \%)$ among other gastro-intestinal nematodes. FECRT interpretation with low eggs per gram of feces associated with this persistence and its relationship with the annual presence of the species in sheep are discussed.

Keywords: Sheep - Marshallagia marshalli - Helminth Resistance to anthelmintics - Algeria.
15. McLEOD R.S., 1995. Cost of major parasites to the Australian livestock industries. Int. J. Parasitol., 25: 1363-1367.

16. MESSOMO NDJANA F., 2006. Etude de la distribution et de la qualité des médicaments vétérinaires au Cameroun. Thèse Doct. vét. Eismv, Dakar, Sénégal, 114 p.

17. ORIPOV A.O., 1984. Host parasite relationships and aspects of pathogenesis in experimental trichostrongyle infections of sheep. Trudy Vses. Inst.Gel'mintol. K.I. Skryabina, 27: 97-103.

18. POWERS K., WOOD L., ECKERT J., GIBSON T., SMITH H., 1982. Guidelines for evaluating the efficacy of anthelminthics in ruminants (bovine and ovine). Vet. Parasitol., 10: 205-264.

19. PRESIDENTE P.J.A., 1985. Methods for detection of resistance to anthelmintics. In: Anderson N., Waller P.J. Eds, Resistance in nematodes to anthelmintic drugs. Glebe, NSW, Australia, CSIRO Division of Animal Health, p. 13-28.

20. URQUHART G.M., ARMOUR J., DUNCAN J.L., DUNN A.M. JENNING F.W., 1996. Veterinary parasitology, 2nd Ed. London, UK Blackwell Science, p. 276-277.

21. VAN SCHALKWYK P.C., GEYSER T.L., RECIO M., ERASMUS F.P. 1979. The anthelmintic efficacy of albendazole against gastrointestinal roundworms, tapeworms, lungworms and liverflukes in sheep. J. S. Afr. Vet. Assoc., 50: 31-35.

22. VAN WYK J.A., MALAN F.S., 1988. Resistance of field strain of Haemonchus contortus to ivermectin, closantel, rafoxanide and benzimidazole in South Africa. Vet. Rec., 123: 226-227.

23. WESCOTT R.B., LEAMASTER B.R., 1982. Efficacy of ivermectin against naturally acquired and experimentally induced nematode infections in sheep. Am. J. vet. Res., $43: 531-533$.

Accepté le 16.03.2012

\section{Resumen}

Boulkaboul A., Boucif A., Senouci K. Determinación de la resistencia de los estróngilos a los anti-helmínticos en el cordero en Argelia

Se llevó a cabo un estudio de la resistencia a los anti-helmínticos de los estróngilos gastrointestinales en el cordero, en una región semi árida de Algeria. Se examinaron en 14 fincas dos anti-helminticos (albendazol e ivermectina), comúnmente utilizados, en 120 y 40 animales respectivamente, para el test de reducción de excreción fecal de huevos (FECRT). Los resultados revelaron una gran eficacidad de la ivermectina (> 95\%) a dosis de $0,2 \mathrm{mg} / \mathrm{kg}$ de peso vivo. La eficacidad global del albendazol a dosis de $5 \mathrm{mg} / \mathrm{kg}$ de peso vivo fue de $94,2 \%$ en cuatro a cinco fincas sobre diez y la reducción de la excreción fecal de huevos estuvo comprendida entre 83,3\% y 100\%. La persistencia de Marshallagia marshalli,después del tratamiento, fue significativamente la más fuerte $(70,4 \%)$ entre otros estrógilos gastrointestinales. Se analiza la interpretación del FECRT con un bajo número de huevos por gramo de heces en relación con esta persistencia y su relación con la presencia anual de la especie en los ovinos.

Palabras clave: Ovino - Marshallagia marshalli - Helminto Resistancia a los antihelmínticos - Argelia. 
